\title{
Spatial Morphological Characteristics of the Yellow River Culture and Amorous Feelings in Lanzhou City based on Fractal Method
}

\author{
Wei-Min GONG ${ }^{a}$, Zhi-Bin ZHANG ${ }^{\text {b, }}$, Xiao-Lu LI \\ Northwest Normal University, Lanzhou, China

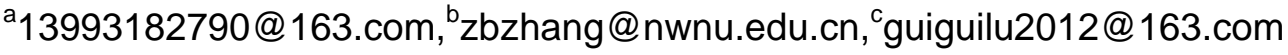

Keywords: Fractal, Riverside recreation, Spatial morphology, Lanzhou City.

\begin{abstract}
Based on the Fractal method, this paper chooses the random aggregation dimension, the spatial correlation dimension and the three dimension indices of the grid dimension to analyze the spatial morphological characteristics of the Riverside Recreational Scenic Area (point) of the Yellow River in the main urban area of Lanzhou city. The results show that: The Yellow River culture style Riverside recreation Scenic Spot (point) system structure has obvious fractal characteristics, at the same time, it has the tendency of self-organization optimization of spatial structure. The random aggregation fractal structure of the Riverside Recreation Scenic Spot (point) system in Lanzhou is a simple single fractal structure. The distribution probability of scenic spot (point) in different scales is large, and the spatial layout is unbalanced.
\end{abstract}

\section{Introduction}

In the time when the world attaches great importance to the ecological environment, the relationship between city and water is highlighted, the development of urban waterfront has become a focus of urban research, in which, the development of waterfront recreational space as a part of urban waterfront space development has attracted people's attention [1]. Many scholars at home and abroad have done a lot of research on the types of waterfront recreational activities [2], the rich riverside recreational activities to develop the image of "River City" [3], Riverside recreational Natural resources [4-6] and human resource [7-9] attraction.

In recent years, with the increasing attention of urban planners to urban waterfront area development, urban waterfront recreation has gradually become an indispensable part of urban tourism projects, so the research on the riverside recreational belt of the Yellow River in Lanzhou is typical, It is an important support to the spatial morphological structure of riverside recreational sights and the development and construction of riverside recreational space in river valley cities. Based on the Fractal method, this paper reveals the spatial morphological features of the Riverside Recreational Scenic Area (point) in the Yellow River culture of Lanzhou city, and gives some feasible suggestions for optimizing the spatial structure.

\section{Research Area and Research Methods}

\section{Research Area}

Lanzhou city, as the capital city of Gansu Province, is not only one of the important gateways on the ancient Silk Road, but also an important historic town in the upper reaches of the Yellow River. From west to east, reach total length of $44.8 \mathrm{~km}$, the annual average runoff is 30.843 billion M3.The main part of the Yellow River, cross-strait vegetation and tidal flat wetlands for Lanzhou city has an extraordinary ecological significance and value, is the most important in Lanzhou Ecological corridor .

\section{Research Methods}

\section{Random Aggregation Dimension}

Considering the variation of the unit value of the radius affects the fractal dimension, the average 
radius is calculated, and the mean radius is:

$$
R_{S} \equiv\left\langle\left(\frac{1}{S} \sum_{\mathrm{i}=1}^{S} \mathrm{r}_{\mathrm{i}}^{2}\right)^{\frac{1}{2}}\right\rangle
$$

There are generally fractal relationships:

$$
R_{S} \propto S^{\frac{1}{D}}
$$

Where $R_{S}$ is the average radius, $R_{i}$ is the first attraction to the central attraction of the European Distance (center of gravity distance), $\mathrm{S}$ is the number of attractions, $\mathrm{D}$ is fractal dimension.

\section{Dimension of Spatial Correlation}

The research on the distribution characteristics of the recreational scenic spots is done in a two-dimensional space, combining the basic definition of the correlation dimension, the calculation of the dimension can be simplified as follows:

$$
C_{(\mathrm{r})} \frac{1}{N^{2}} \sum_{\mathrm{i}, j=1}^{N} H\left(r-\mathrm{d}_{\mathrm{ij}}\right)
$$

In this formula, $\mathrm{R}$ is a yardstick (yardstick), $\mathrm{D}_{\mathrm{ij}}$ is the Euclidean distance of the $\mathrm{i}, \mathrm{j}$ two scenic spots (points), that is, the crow distance (crow distance), h (x) is the Heaviside step function, namely:

$$
C(\lambda r) \propto \lambda^{\alpha} C(r) \quad C(r) \propto \gamma^{\alpha}
$$

$\alpha=D$ is fractal dimension, also called spatial correlation dimension. According to the spatial distribution pattern, there are similarities between the definition of spatial correlation dimension and grid dimension, they are to a certain extent to reflect the equilibrium characteristics of the spatial distribution of recreational scenic spots (dots), and also embody the relevance and accessibility of scenic spots and scenic spots (dots).

\section{Research Object and Data Source}

In view of the lack of uniform and standard recreational resources norms in China, this paper chooses the "People's Republic of China Tourism Resources Classification, investigation and Evaluation" (GBT18972-2003) as the basis for evaluation, in the Yellow River culture zone along the scenic spots (points) on the basis of a total of 55 scenic spots (points). Measuring means using ArcGIS software to map the Yellow River culture in the main urban areas of Lanzhou City scenic spots (point) space distribution map, scenic spots (point) positioning with GPS data mainly, and with the help of Baidu Map geographical coordinates acquisition and precision proof detection, scenic spots (points) of the space positioning accuracy has been certain protection, The rest of the data statistical analysis are implemented by SPSS software.

\section{Fractal of Spatial Structure of Scenic Spots (Point) System in the Yellow River Culture Zone of Lanzhou City}

\section{Spatial Agglomeration of Recreational Scenic Spots (Point) System}

A survey of the Yellow River culture in the main city of Lanzhou, with the interview and questionnaire of the scenic spots (points), the results show that Zhongshan Bridge, Baita Park, Yellow River mother sculpture and Water Tanker Expo Park, the Yellow River cultural amorous feelings with well-known scenic spots (points) comprehensive ranking of the top four, taking into account The comprehensive influence of tourism resources and the significance of cultural representation, this paper chooses Zhongshan Bridge as the core scenic Spot (point) of random aggregation dimension to calculate the number of scenic spots (points) in the radius range by 
gyration, and then obtains the corresponding number of tourist attractions by changing the $\mathrm{R}$ value, then according to the obtained Point column (ri, S) The corresponding average RADIUS RS is computed, the point column (ln s,ln RS) is plotted in the rectangular coordinate system, the linear regression analysis is carried out, and finally the double logarithm statistic chart of the aggregation fractal dimension is obtained (Fig 1).

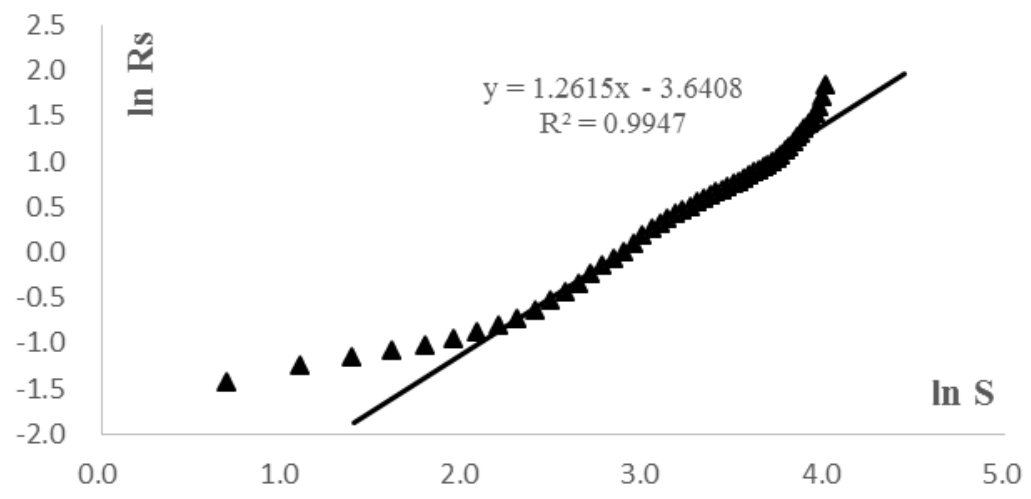

Fig.1The log-log plot for the aggregation dimension of recreation spots system in Lanzhou

As shown in Fig. 1, most of the points are linearly distributed, with obvious scale-free, and the scale-free region is wide, which indicates that the random aggregation fractal structure of the Riverside Recreational Scenic Spot (point) system in Lanzhou is a simple and single fractal structure. After regression analysis of the coordinate points in the scale-free region, the discriminant coefficients of the regression equation are $\mathrm{r} 2=0.9974$, which shows that $99.74 \%$ of the variable RS changes with S change in the process of variation, and the fitting degree is very high. The Yellow River culture of Lanzhou city with recreational scenic spot (point) system fractal dimension value is $0.7927<2$, it shows that the spatial structure of the whole system has strong stochastic aggregation, and the core scenic spots in the recreation belt consciously become the center of the system's self evolution, the whole system is in the mature stage of the condensed model of the limited diffusion group, and the density of scenic spots (dots) from the core (point) gradually descending to the perimeter, in line with the law of distance attenuation, is a normal aggregation state.

\section{Spatial Correlation of Recreational Scenic Spots (Point) System}

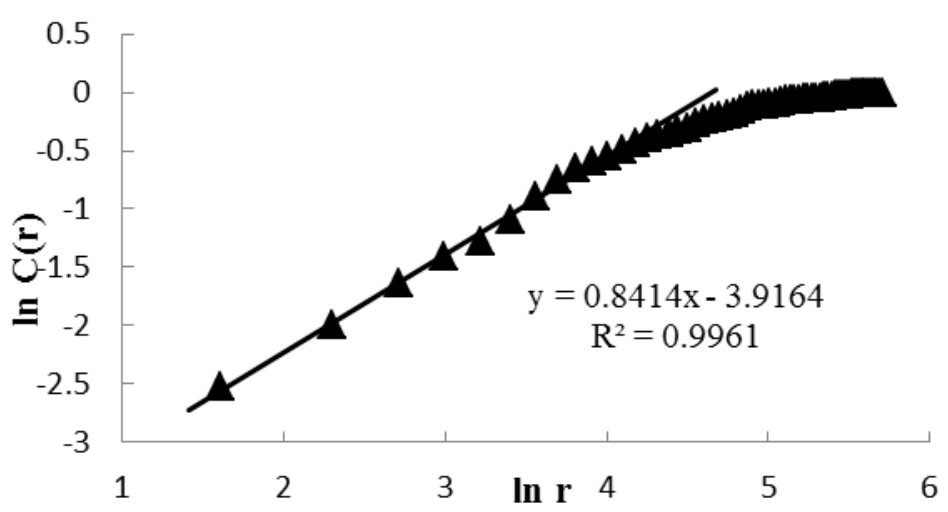

Fig.2 The log-log plot for the correlation dimension of Lanzhou Yellow River recreation spots systems

From Figure 2 can be seen, Lanzhou Yellow River cultural amorous feelings with recreational scenic spots (point) system in a certain size segmentation area has a certain scale-free, scenic spots (points) of the spatial structure is fractal state. From table 2, the Yellow River culture in Lanzhou city with recreational scenic spots (point) the spatial correlation dimension of the system is 
$0.8414, \mathrm{~d}<1$, which indicates that the riverside recreational Scenic Spot (point) is densely distributed in a certain area, which accords with the actual situation of the Riverside Recreational Scenic Spot (point) which is mainly distributed in the main urban Most scenic spots (dots) are densely distributed in the downstream areas of the Yellow River in the eastern part of the city, and the European distance between them is small, while in the upper reaches of the Yellow River in the western part of the city, the number of recreational scenic spots (dots) is sparse. With the actual investigation, the scenic spots (points) in the western area of the recreation belt are sparse and the quality of recreational resources is low, the surrounding comprehensive service facilities are underdeveloped, and the diversion of passenger flow is weak in the middle reaches of the recreation system.

\section{Spatial Distribution Balance of Recreational Scenic Spot (Point) System}

According to the calculation method and formula of grid dimension, using ArcGIS, the rectangular region of the Yellow River Culture zone which is studied in this paper is divided into several different meshes, and the calculation data of the grid dimension of the Yellow River Culture Zone (point) system in Lanzhou are calculated $\mathrm{N}(\varepsilon)$ and I $(\varepsilon)$ (table 1), Based on the data plotting of the double logarithmic coordinate diagram (Fig 3), the capacity dimension D0 and Information Dimension D1 (table 2) of the system of the Yellow River cultural amorous feelings in Lanzhou City are analyzed.

Table.1 The determining data of grid dimension of Lanzhou Yellow River recreation spots system

\begin{tabular}{|c|c|c|c|c|c|c|c|c|c|}
\hline $\mathrm{K}$ & 2 & 3 & 4 & 5 & 6 & 8 & 10 & 15 & 20 \\
\hline $\mathrm{N}(\varepsilon)$ & 3 & 6 & 7 & 10 & 10 & 15 & 17 & 20 & 25 \\
\hline $\mathrm{I}(\varepsilon)$ & 0.6315 & 1.4484 & 1.5703 & 1.6637 & 2.0609 & 2.1279 & 2.3521 & 2.6501 & 2.9907 \\
\hline $\mathrm{K}$ & 25 & 30 & 40 & 60 & 80 & 100 & 120 & 150 & 200 \\
\hline $\mathrm{N}(\varepsilon)$ & 29 & 34 & 35 & 40 & 46 & 46 & 47 & 49 & 51 \\
\hline $\mathrm{I}(\varepsilon)$ & 3.1200 & 3.2730 & 3.3531 & 3.5490 & 3.7458 & 3.7551 & 3.7962 & 3.8561 & 3.9065 \\
\hline
\end{tabular}

As can be seen from fig. 3, within a certain scale of measurement, the Yellow River cultural amorous feelings in Lanzhou city show the fractal structure characteristic, but because the riverside recreational space belongs to the long strip, some information dimension and the capacity dimension are unstable, but after repeated testing the validity of the mesh segmentation, The test results conform to the theoretical significance and the actual situation. As shown in table 5, the Yellow River culture of Lanzhou city with recreational scenic spots (point) system grid capacity dimension is 0.9318 , the information dimension is 0.8240 , two values are less than 1 and there are some differences between them, which shows that the probability of the distribution of scenic spots in different scales is large and the spatial layout is unbalanced.

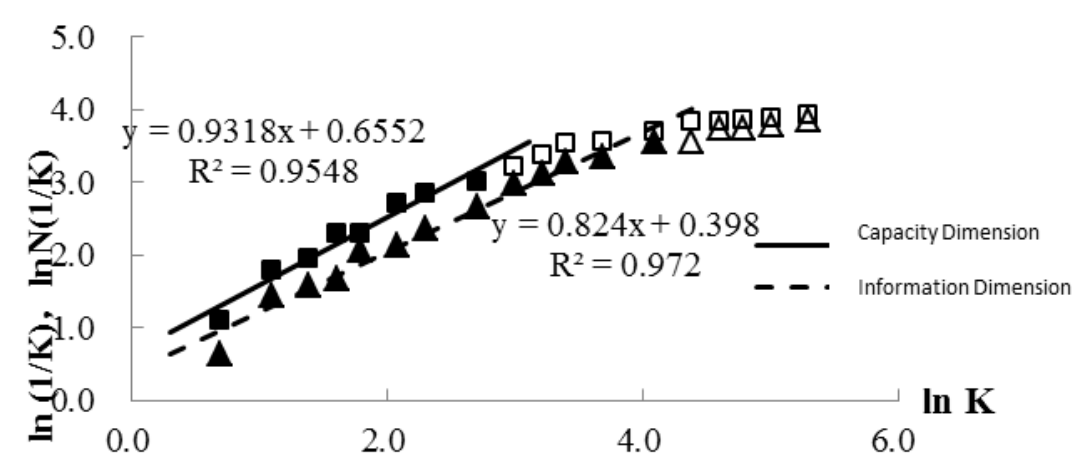

Fig.3 The log-log plot for the grid dimension of Lanzhou Yellow River recreation spots system 
The grid capacity dimension is approaching 1 (table 2), indicating that the distribution of recreational scenic spots (dots) is in a line (e.g. railways, highways, rivers, coasts and so on), this and the reality of Riverside Recreational Scenic Spot (point) is along the Yellow River across the belt distribution of the situation coincide, something narrow "valley type" The urban structure exerts a subtle influence on the spatial structure of the scenic spots (points) of the whole Yellow River culture. The information dimension value 0.8240 indicates that there is a significant aggregation feature in the whole riverfront open belt, and it is not uniform layout.

Table.2 The values of fractal dimension of Lanzhou Yellow River recreation spots system

\begin{tabular}{|c|c|c|c|c|c|}
\hline \multicolumn{2}{|c|}{ Dimension of spatial correlation } & \multicolumn{2}{c|}{ Dimension of grid capacity } & \multicolumn{2}{c|}{ Grid information Dimension } \\
\hline $\mathrm{D}$ & $\mathrm{R}^{2}$ & $\mathrm{D}_{0}$ & $\mathrm{R}^{2}$ & $\mathrm{D}_{1}$ & $\mathrm{R}^{2}$ \\
\hline 0.8414 & 0.9961 & 0.9318 & 0.9548 & 0.8240 & 0.9720 \\
\hline
\end{tabular}

\section{Conclusion}

In this paper, three kinds of fractal dimensions are used to analyze the spatial morphological characteristics of the scenic spots (points) of the Yellow River culture in Lanzhou from different angles and scales. And three methods finally draw the fractal structure of the scenic Spot (point) basically similar, namely the Yellow River culture of Lanzhou city with recreational Scenic Area (point) show around a single core scenic spot.

The long and narrow city form of Lanzhou is leading to the Yellow River cultural amorous feelings scenic spots (points) the spatial distribution has the number of scenic spots (points) east and west less, the structure of the nishimatsu of the single core attractions of the Ribbon space features the main reason.

According to the research process and results, it is feasible and meaningful to study the spatial morphological characteristics of riverside recreational space Scenic Spot (point) system by fractal correlation theory and fractal dimension quantification, and the empirical evidence shows that the structure of Riverside Recreation Area (point) space has fractal characteristics, It also provides theoretical guidance and scientific evaluation criteria for the future use of fractal theory to urban recreational space planning and construction and even urban overall planning.

\section{Reference}

[1] Saunders, Paul R., Senter, Herman P., Jarvis, James P. Forecasting recreation on demand in the upper Savannah River Basin[J].Annals of Tourism Research, 1981, 9(2): 236-250.

[2] Rice, G., Urban , M.A. Where is River City, USA? Measuring community attachment to the Mississippi and Missouri Rivers[J].Journal of Cultural Geography, 2006, 24(1): 1-34.

[3] Arnould, E.J., Price, L.L. River magic: Extraordinary experience and the extended service encounter [J].Journal of Consumer Research, 1993, 20(6): 24-45.

[4] Ndiaye, A.A practitioner's view of conservation and development in Africa integrated management and the Djoudj National Park in Senegal[J].Africa Today, 2001, 48(1): 100-109.

[5] Shrestha, R.K., Stein, T.V. Valuing nature-based recreation in public natural areas of the Apalachicola River region, Florida[J].Journal of Environmental Management, 2007, 85: 977-985.

[6] Josef, S.River related tourism in Europe_—an overview[J].Geographical Journal, 1995, 35(4): 443-458.

[7] Savage, V.R., Shirlena, Huang, Chang, T.C. The Singapore River thematic zone sustainable tourism in an urban context[J].The Geographical Journal, 2004,170(3): 212-225.

[8] Chang, T.C., Shirlena, Huang, Recreating place, replacing memory Creative destruction at the 
Singapore River[J].Asia Pacific Viewpoint, 2005, 46(3): 267-280.

[9] Fisher, D, G. The potential for rural heritage tourism in the Clarence Valley of Northern New South Wales[J].Australian Geographer, 2006, 37(3): 411-424. 